\title{
Additive Effects on Thermotropic Liquid Crystal Polymer Alloys II. Effects of Third Component of Thermotropic Liquid Crystal Polymer on Mechanical Properties of Vectra A-Reinforced Poly(butylene terephthalate)
}

\author{
Li-Min Sun,* Takeya Sakamoto, Shigeyuki Ueta, \\ Keiko Koga, and Motowo TaKaYANagi** \\ Department of Industrial Chemistry, Faculty of Engineering, Kyushu Sangyo University, \\ Matsukadai, Higashi-ku, Fukuoka 813, Japan
}

(Received February 7, 1994)

\begin{abstract}
The effects of addition of the 3rd component of thermotropic liquid crystal polymer (TLCP) on the mechanical properties and structure of Vectra A-reinforced poly(butylene terephthalate) (PBT) were studied. The 3rd TLCP was the copolymer based on $p$-hydroxybenzoate (PHB) and ethylene terephthalate, known as X-7G. PBT, Vectra A, and the third TLCP were blended in the molten state, the extrudate of which was cut and injection-molded. The mechanical properties of the blends were improved by the combination of the two kinds of TLCP. The dynamic viscoelastic analysis indicated that the thermal endurance of the ternary blends were enhanced when Vectra A was used as a major TLCP reinforcement. SEM revealed that both the binary and ternary blends were phase-separated, and the introduction of the 3rd component of TLCP was profitable for TLCP to form finer fibrils at the skin part of the sample. X-Ray diffraction analysis showed that the orientation of Vectra A fibril in the blend was raised by the addition of the 3rd TLCP, resulting in the improvement of the mechanical properties of the ternary blends. The conclusions obtained in this paper agreed with those reported in our previous paper.
\end{abstract}

KEY WORDS Poly(butylene terephthalate) / Thermotropic Liquid Crystal

/ Ternary Blends / Mechanical Properties / Morphology / X-Ray Diffraction Analysis /

In our previous paper, ${ }^{1}$ we reported the effects of the 3rd component of TLCP on the mechanical properties of Vectra A (VA)reinforced polycarbonate (PC). The mechanical properties of PC/VA binary blend were improved dramatically by addition of a small amount of the 3rd component of TLCP composed of PHB and PET, which was a X-7G type semi-aromatic TLCP, Rodlan of the trade name of Unitika Co. The effects were ascribed to the enhancement of orientation of TLCP ( mainly VA) in fibril, which was supported by the X-ray diffraction and the theoretical analysis based on a two-phase blend model and the SEM observation.

In this paper, we continued to combine the both advantages of Vectra A and Rodlan and blended them with the matrix resin of poly(butylene terephthalate) (PBT), forming a ternary blend system. Kimura and Porter ${ }^{2}$ reported the thermal properties and the dynamic mechanical measurements of the binary blend of $\mathrm{PBT} / \mathrm{X}-7 \mathrm{G}$ prepared by a solution precipitation method and found that both components were compatible due to the molecular interaction between PBT and the

* On Leave from the Department of Applied Chemistry, Shanghai Jiao Tong University, Shanghai 200030, China.

** To whom all corespondences should be addressed. 
"flexible" low- $T_{\mathrm{g}}$ segment of X-7G. Kiss ${ }^{3}$ studied the binary blends of Hoechst-Celanese Vectra with a series of engineering plastics including PBT prepared by a melt blending method, and found that liquid crystal domains of VA showed a "gnarled" appearance in PBT matrix by SEM. There were also other papers $^{4-7}$ dealing with the PBT/VA or PBT/X-7G binary blend systems. From these papers, it was found that the binary blend of $\mathrm{PBT} / \mathrm{X}-7 \mathrm{G}$ was partial compatible and the binary blend of PBT/VA was incompatible. Actually, judging from the criteria presented in our previous paper ${ }^{1}$ their mechanical properties and thermal endurance were not good. We expected in this paper that the mechanical properties of the binary blend of PBT/TLCP could be improved by use of the combination of two kinds of TLCP of Vectra A and Rodlan.

\section{EXPERIMENTAL}

\section{Materials}

Poly(butylene terephthalate) (PBT) was supplied from Toray Co. A wholly aromatic TLCP (Vectra A 950, abbreviated by VA) based on copolycondensation of $p$-hydroxybenzoic acid (PHB) and 2,6-hydroxynaphthioc acid (HNA) was supplied by Polyplastics Co. The semi-aromatic TLCPs based on the copolymer of PET and PHB (Rodlan LC 3000 and Rodlan LC 5000, abbreviated by R3 and R5, respectively) were supplied by Unitika Co. R5 had a higher content of PHB composition. Table I lists the characteristics of these materials.

\section{Preparation of Blends}

All the materials were dried under vacuum at $100^{\circ} \mathrm{C}$ for at least $24 \mathrm{~h}$ before used. The blends were prepared by using a Mini Max Polymer Evaluation System of Custom Scientific Instrument Inc. The blended samples were prepared by shearing followed by extrusion. The bar specimens for tensile testing were prepared by injection-molding. The temperatures of the rotor and the spinning die for extrusion were $250^{\circ} \mathrm{C}$ and $310^{\circ} \mathrm{C}$, respectively. The temperature of the cylinder and the mold for injection were $310^{\circ} \mathrm{C}$ and $100^{\circ} \mathrm{C}$, respectively.

\section{Test Procedures}

Tensile testing was conducted by a Tensilon STM-T50BP of Orientec Co. The elongation rate was kept at $10 \mathrm{~mm} \mathrm{~min}^{-1}$. The dimension of a bar specimen was $1 \times 2.5 \times 50 \mathrm{~mm}$. The

Table I. Characteristics of polymers used in this paper

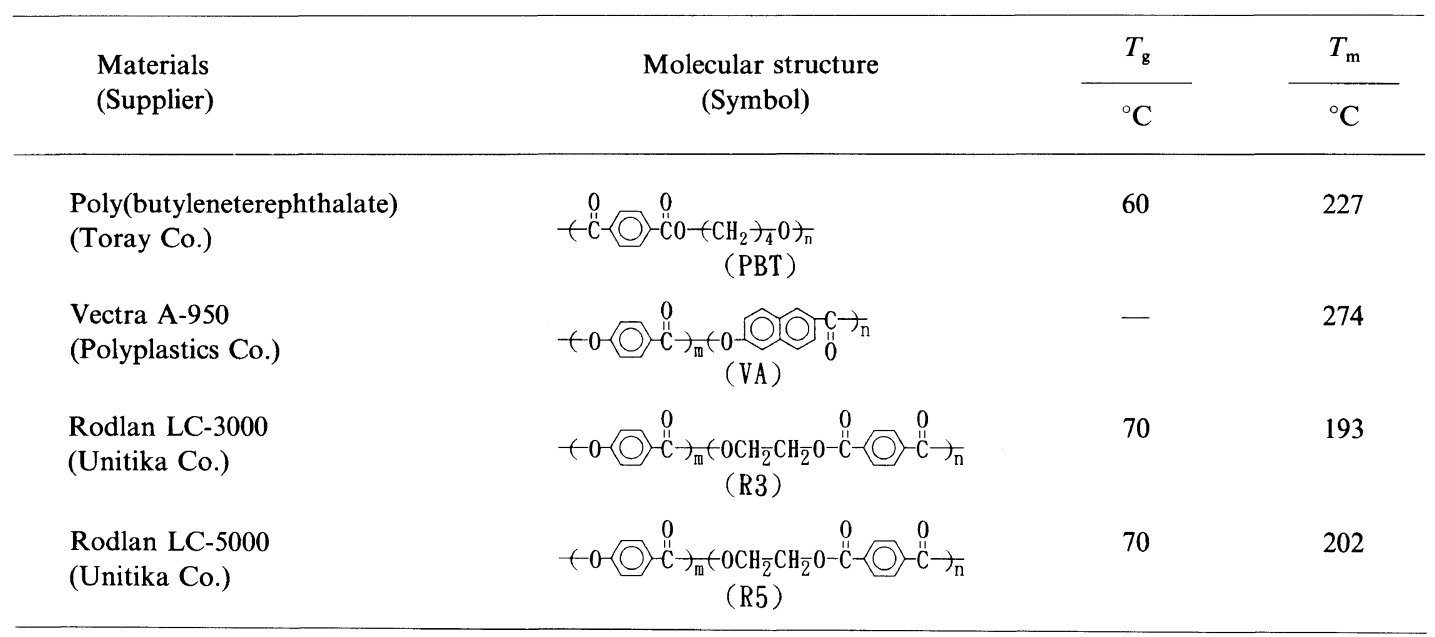


same specimen was used to measure the dynamic viscoelasticity in a Rheovibron of Orientec Co. at $110 \mathrm{~Hz}$ over a temperature range from room temperature to $300^{\circ} \mathrm{C}$. The morphology of the fractured surface of an injection-molded bar specimen (broken in liquid nitrigon) was examined in a Hitachi S-510 Scanning Electron Microscope (SEM). The X-ray diffraction was carried out in a Rigaku CN 2013 Diffractometer to compare the orientation of TLCP in the injectionmolded sample. The wide angle $\mathrm{X}$-ray diffraction (WAXD) pattern was taken by a flat-plate camera.

\section{RESULTS AND DISCUSSION}

\section{Mechanical Properties}

The tensile mechanical properties of the binary blends of PBT with three kinds of TLCPs were firstly tested. The ultimate strengths and initial moduli vs. the weight ratios of TLCP to PBT for the binary blends of PBT/VA, PBT/R5, and PBT/R3 are shown in Figures 1-a, 1-b, and 1-c, respectively. Judging from the criteria presented in our previous paper, ${ }^{1}$ the mechanical properties of PBT/VA (Figure 1-a) were quite poor, being largely lower than the upper bound of modulus for the two-phase blend. For PBT/R5 (Figure 1-b), the moduli-composition curve approched the upper bound and showed improved mechanical properties compared with PBT/ VA. The mechanical properties of PBT/R3 (Figure 1-c) surmounted the upper bound of modulus and showed an excellent case.

The results above revealed that the affinity between PBT and TLCP exerted influence on the mechanical properties of their blends, depending on the chemical structure of TLCP. R3 possessed relatively higher content of flexible component (PET) and showed a stronger affinity to $\mathrm{PBT}$, resulting in better mechanical properties of the blend. PBT/VA was a reversed case and PBT/R5 was situated between the above two cases.
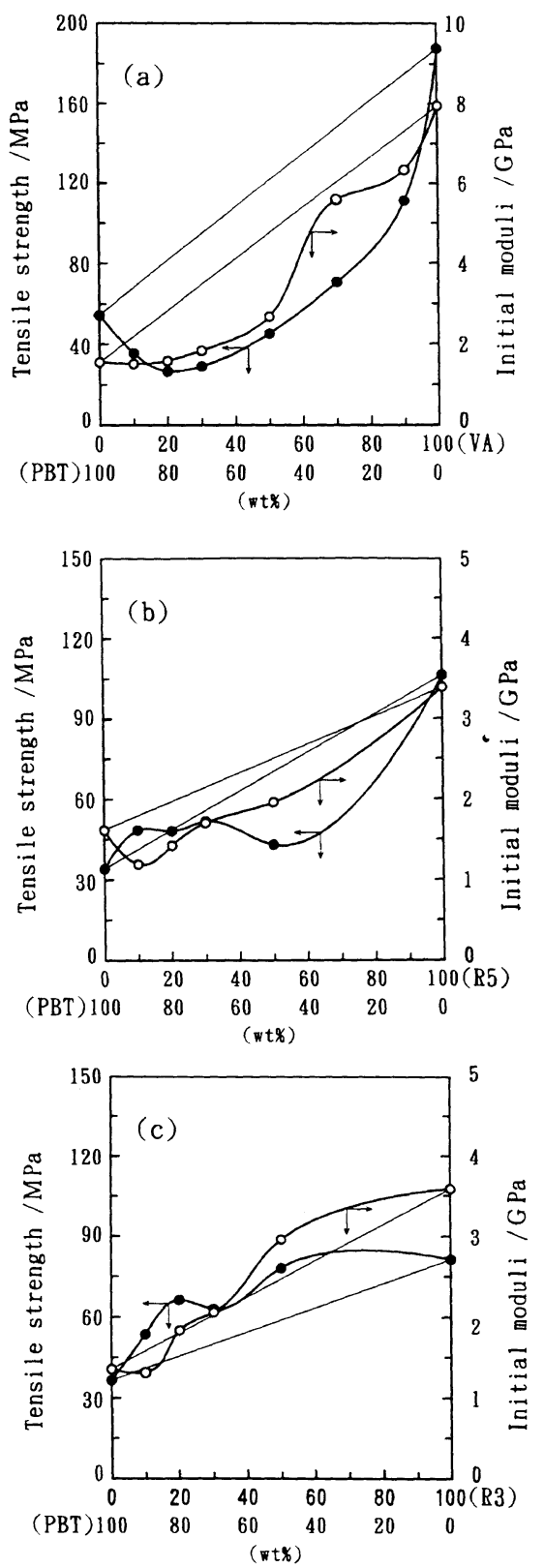

Figure 1. Tensile strength and initial modulus $v s$. TLCP content (wt \%) in the binary blends: (a) PBT/VA, (b) $\mathrm{PBT} / \mathrm{R} 5$, and (c) PBT/R3.

However, VA was hoped to be a main reinforcement in PBT because of its higher mechanical properties and thermal endurance than that of semi-aromatic R3 or R5. The 

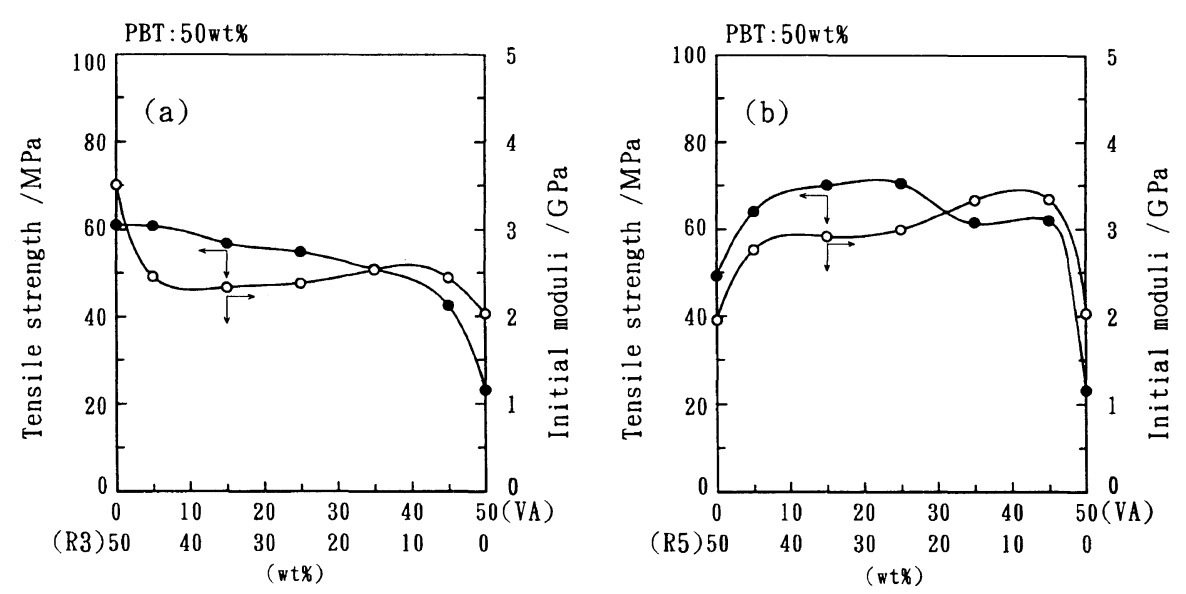

Figure 2. Tensile strength and initial modulus $v s$. TLCP content (wt \%) composed of R and VA in the ternary blends for the PBT content wt $\%=50 \%$ : (a) PBT/R3/VA and (b) PBT/R5/VA.

combination of VA with $\mathrm{R} 3$ or $\mathrm{R} 5$ as a reinforcement in a ternary system was expected to be a possible way for achieving both better mechanical properties and higher thermal endurance. Thus, it was necessary to survey the compatibility of two kinds of TLCP, VA/R3, or VA/R5, which had been studied in our previous paper ${ }^{1}$. It was shown that the blend of VA/R5 was better to follow the volume additivity of modulus than VA/R3, although the moduli in both case were lower than the upper bounds of the moduli. The results were reasonable as $\mathrm{R} 5$ possessed more aromatic nature than $\mathrm{R} 3$, and resulted in strong affinity to the wholly aromatic TLCP of VA.

For the ternary blends, the content of PBT was kept at $50 \mathrm{wt} \%$ and the ratio of VA to R3 or R5 was changed. Figures 2-a and 2-b show the ultimate strenghs and initial moduli of the ternary blends $v s$. the ratios of VA to R3 and $\mathrm{R} 5$, respectively. The mechanical properties of the ternary blend of PBT/R3/VA (Figure 2-a) did not show a remarkable variation over a wide range of R3/VA at $50 \mathrm{wt} \%$ of TLCP. On the other hand, for the PBT/R5/VA ternary blend, the improved mechanical properties were found over a wide range of ratios of R5 to VA, especially at PBT/R5/VA=5/0.5/4.5. Apparently, R5 was more effective than R3

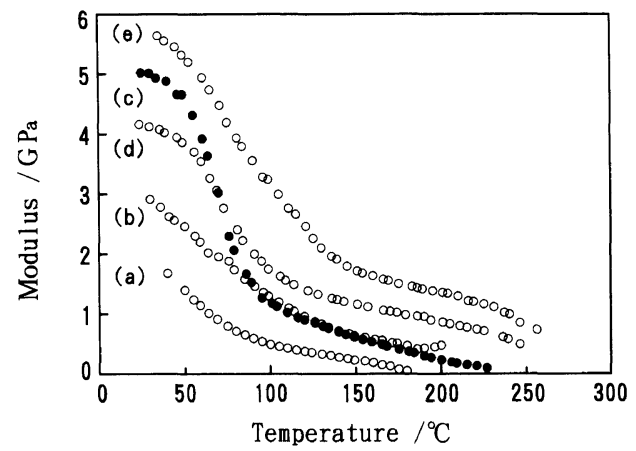

Figure 3. Tensile storage modulus vs. temperature: (a) PBT, (b) PBT $/ \mathrm{VA}=5 / 5$, (c) $\mathrm{PBT} / \mathrm{R} 3=5 / 5$, (d) $\mathrm{PBT} / \mathrm{R} 5=$ $5 / 5$, and (e) $\mathrm{PBT} / \mathrm{R} 5 / \mathrm{VA}=5 / 0.5 / 4.5$.

to improve the mechanical properties of TLCP reinforced PBT. The reason for it might be that R5 had a balanced affinity to both PBT and VA.

\section{Dynamic Viscoelasticity}

Figure 3 shows the dynamic storage moduli of binary and ternary blends in which the content of TLCP were kept at $50 \mathrm{wt} \%$. For comparison, the dynamic storage modulus of PBT is also shown in Figure 3 (curve a). Corresponding to the result of tensile testing, the modulus of binary blend of PBT/VA (curve b) was quite low. On the other hand, at room 
temperature the modulus of binary blend of PBT/R3 (curve c) was high, but it decreased gradually toward the high temperature side and remarkably fell around $70^{\circ} \mathrm{C}$. The transition region around $70^{\circ} \mathrm{C}$ was apparently associated with the primary relaxation of PET segment of R3. The same trend was also observed in PBT/R5 binary blend (curve d), although the relaxation became mild and the decrease of the modulus toward the high temperature side was not so rapid as that of PBT/R3. For the ternary blend of $\mathrm{PBT} / \mathrm{R} 5 / \mathrm{VA}=5 / 0.5 / 4.5$ (curve e), the modulus was almost the same with that of $\mathrm{PBT} / \mathrm{R} 3=5 / 5$ at room temperature, but with the increasing temperature, the former showed a slower change, and after $70^{\circ} \mathrm{C}$ it maintained a higher value than the latter. Thus, it could be concluded that the combination of VA and a small amount of R5 was useful for the improvement of modulus and thermal endurance in comparison with the binary blend of PBT/VA.

\section{SEM}

Figure 4 shows the SEM morphology of the core part of the injection-molded sample of $\mathrm{PBT} / \mathrm{VA}=5 / 5$. The domain of VA was not well developed and showed a shape of elongated sphere. VA could not play a role of reinforcement in such a state, resulting in poor mechanical properties (refer to Figure 1-a). Skin-core structure could not be discriminated in this sample.

Figure 5 shows the SEM morphology of the injection-molded sample of $\mathrm{PBT} / \mathrm{R} 5=5 / 5$ at core part. The long fibrils lower than $5 \mu \mathrm{m}$ in diameter were found orientating along the flow direction. It could be observed in the fractured surface that the TLCP fibrils were pulled-out and broken. Skin layer was well developed for the PBT/R5 compared with PBT/VA perhaps due to the increase of interfacial affinity between PBT and R5. The mechanical properties of such a system as PBT/R5 were improved (refer to Figure 1-b).

Figures 6-a and 6-b show the SEM

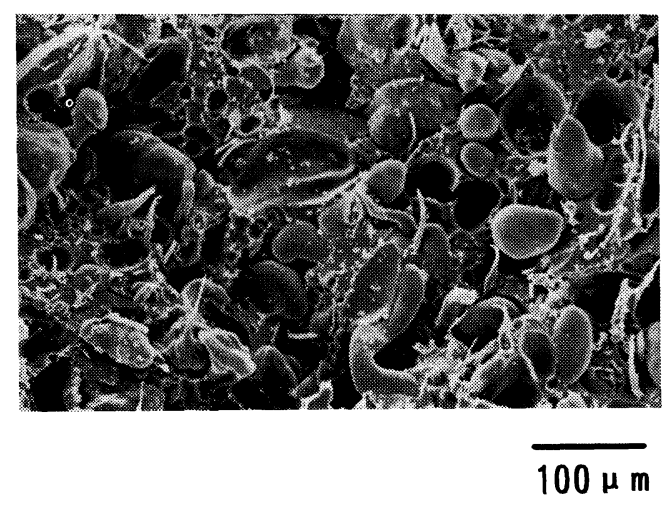

Figure 4. SEM morphology of fractured surface of injection-molded sample of $\mathrm{PBT} / \mathrm{VA}=5 / 5$ at core part.

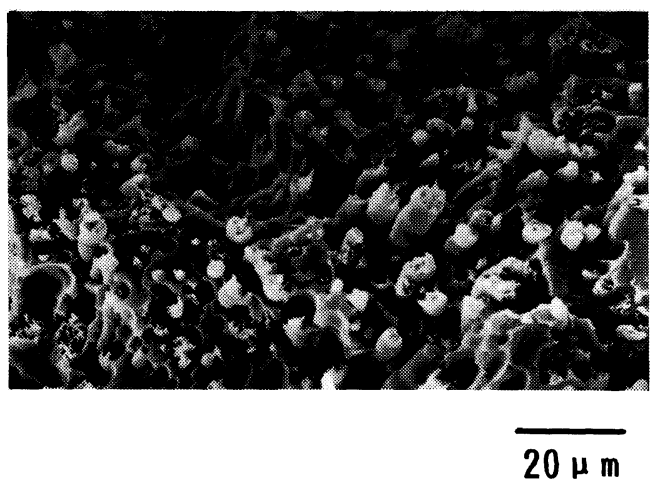

Figure 5. SEM morphology of fractured surface of injection-molded sample of $\mathrm{PBT} / \mathrm{R} 5=5 / 5$ at core part.

morphologies of the injection-molded sample of $\mathrm{PBT} / \mathrm{R} 3=5 / 5$ at the skin and core part, respectively. In the skin part, the TLCP formed long and fine fibrils (diameter was about $0.5 \mu \mathrm{m}$ ), which packed regularly and oriented well along the flow direction. That meant an excellent reinforced structure. But in the core part, no fibril could be observed, showing a quite different feature. Thus, it was reasonable that the skin part of the injection-molded sample played a more important role in giving a better mechanical properties of the blend (refer to Figure 1-c).

Figures 7 and 8 show the SEM morphologies of the injection-molded of $\mathrm{PBT} / \mathrm{R} 3 / \mathrm{VA}=$ 5/0.5/4.5 and PBT/R5/VA $=5 / 0.5 / 4.5$ at the 

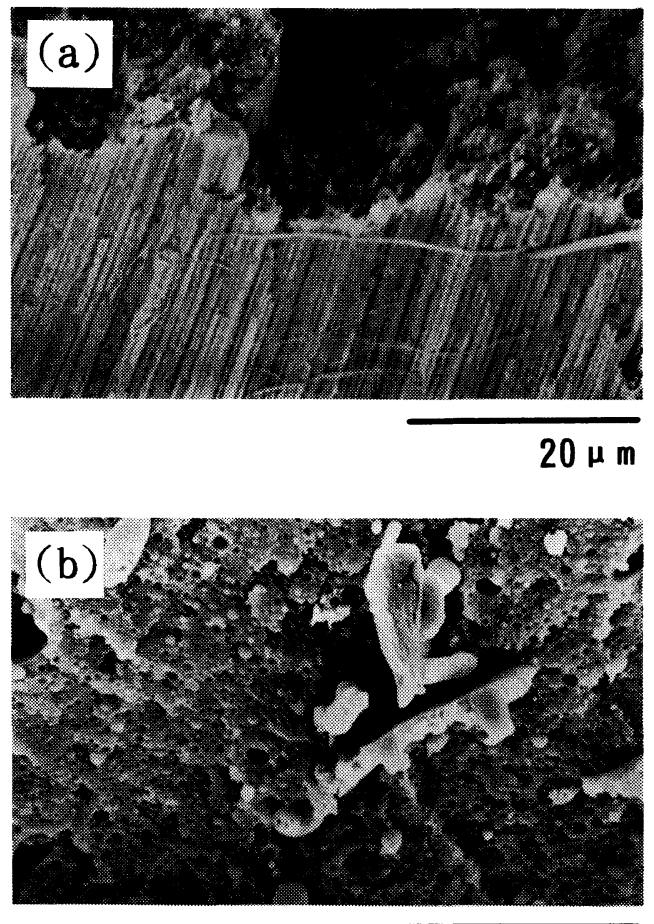

$50 \mu \mathrm{m}$

Figure 6. SEM morphologies of fractured surfaces of injection-molded sample of PBT/R3 = 5/5: (a) at skin part and (b) at core part.

skin part, respectively. There were welldeveloped skin layers in both cases, but the TLCP fibrils formed in the latter case were longer in dimension and higher in orientation than that in the former case. However, the TLCP in the core part showed an irregular, layered structure in both cases. Comparison of Figure $8(\mathrm{PBT} / \mathrm{R} 5 / \mathrm{VA}=5 / 0.5 / 4.5)$ with Figure $4(\mathrm{PBT} / \mathrm{VA}=5 / 5)$ explained the improvement of the mechanical properties in the ternary blend. We discussed in our previous paper that the effects of combination of VA and Rodlan on the mechanical properties were ascribed to the reduction of the interfacial energy through formation of finer fibrils. Similarly the interfacial energy between PBT and the TLCP reinforcement was reduced, resulting in longer and finer TLCP fibrils under a high shear stress during injection-molding and improved me-

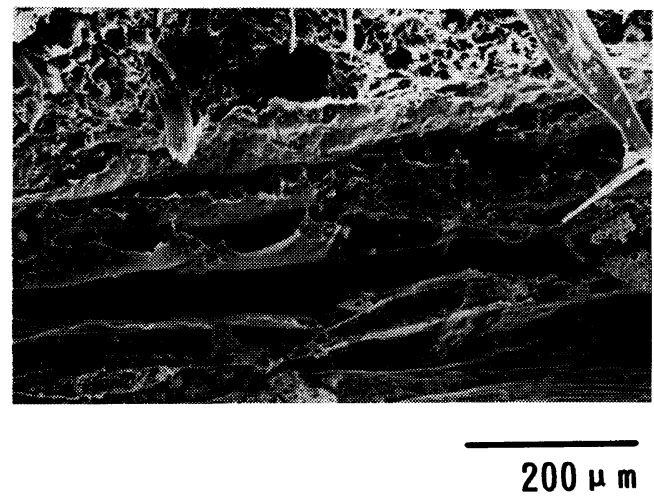

Figure 7. SEM morphology of fractured surface of injection-molded sample of $\mathrm{PBT} / \mathrm{R} 3 / \mathrm{VA}=5 / 0.5 / 4.5$ at skin part.

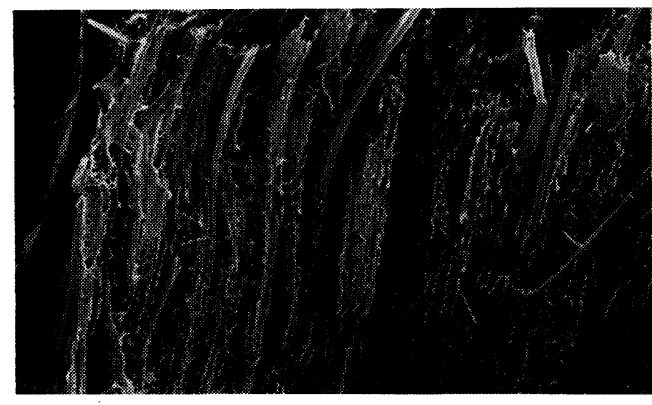

$500 \mu \mathrm{m}$

Figure 8. SEM morphology of fractured surface of injection-molded sample of PBT/R5/VA = 5/0.5/4.5 at skin part.

chanical properties.

\section{$X$-Ray Diffraction}

Figures 9-a, 9-b, and 9-c show the WAXD patterns of the skin part of the injection-molded sample bars of $\mathrm{PBT} / \mathrm{VA}=5 / 5, \mathrm{PBT} / \mathrm{R} 3 / \mathrm{VA}=$ 5/0.5/4.5, and PBT/R5/VA = 5/0.5/4.5, respectively. For comparison, Figures $9-\mathrm{d}$ and 9-e show the WAXD patterns of PBT $/ \mathrm{R} 5=5 / 5$ and $\mathrm{PBT} / \mathrm{R} 3=5 / 5$ for the samples prepared by the same injection molding conditions, respectively. The intensity of the equatorial diffraction of (110) for the injection-molded bar of VA was selected to evaluate the orientation of TLCP in the fibril. The width of half 

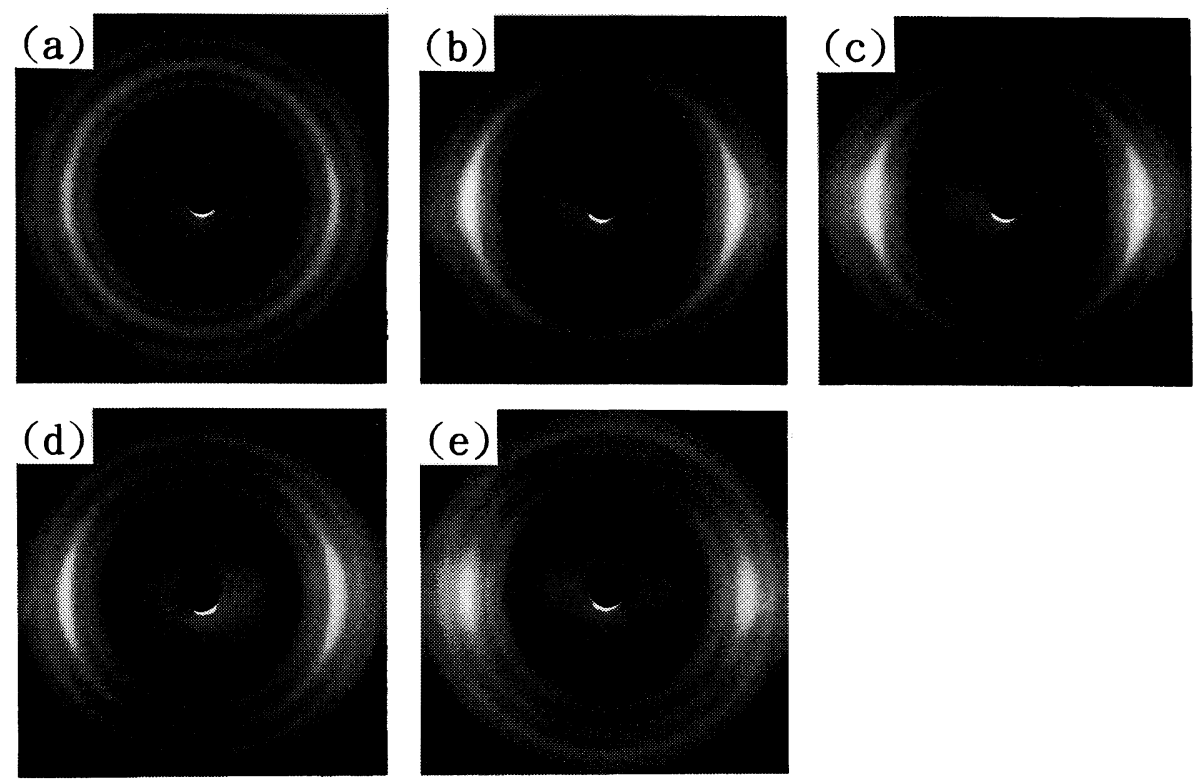

Figure 9. WAXD patterns of injection-molded sample at skin part: (a) $\mathrm{PBT} / \mathrm{VA}=5 / 5$, PBT/R3/VA=5/0.5/4.5, (c) PBT/R5/VA=5/0.5/4.5, (d) PBT/R5=5/5, and (e) PBT/R3=5/5.

Table II. Orientation parameter $(Q \%)$

\begin{tabular}{ccccccc}
\hline Sample & VA & $\begin{array}{c}\text { PBT/VA } \\
5 / 5\end{array}$ & $\begin{array}{c}\text { PBT/R5 } \\
5 / 5\end{array}$ & $\begin{array}{c}\text { PBT/R3 } \\
5 / 5\end{array}$ & $\begin{array}{c}\text { PBT/R3/VA }= \\
5 / 0.5 / 4.5\end{array}$ & $\begin{array}{c}\text { PBT/R5/VA } \\
5 / 0.5 / 4.5\end{array}$ \\
\hline \multirow{2}{*}{$Q / \%$} & 71.1 & 65.5 & 71.7 & 75.6 & 75 & 75.6 \\
\hline
\end{tabular}

maximum intensity was evaluated from an intensity curve scanning along the circumferential direction by use of a photodensitometer. The conventional orientation parameter $Q$ was obtained from the width of half maximum $\theta$ according to the following equation:

$$
Q=[(180-\theta) / 180] \times 100 \%
$$

The results are shown in Table II.

For the binary blends of PBT/VA, PBT/R5, and PBT/R3, the order of orientation parameters corresponded to the order of the mechanical properties, that is, if the degree of orientation was high, for example, for $\mathrm{PBT} / \mathrm{R} 3=5 / 5$, the mechanical properties were also high (refer to Figure 1-c). Comparing $\mathrm{PBT} / \mathrm{VA}=5 / 5$ with $\mathrm{PBT} / \mathrm{R} 5 / \mathrm{VA}=5 / 0.5 / 4.5$, the degree of orientation of VA was enhanced remarkably, resulting in the improvement of the mechanical properties. In the ternary blends, R5 was more effective to enhance the orientation of VA than R3. Following the diagramming method to evaluate the modulus of TLCP in the two-phase blending system presented in our previous paper ${ }^{1}$, the moduli of TLCP in the binary and ternary blends for PBT content $=50 \%$ were obtained. In the binary blend of PBT/VA $=5 / 5$, the extrapolated modulus of VA was only $2.5 \mathrm{GPa}$, largely lower than that of unblended VA, $9.5 \mathrm{GPa}$. On the other hand, in the ternary blend of PBT/R5/VA $=5 / 0.5 / 4.5$, the extrapolated modulus of TLCP was $5.0 \mathrm{GPa}$, which was close to the value of unblended TLCP, 6.0 GPa and one time higher than that of VA. 
From the results above, it could be concluded that the introduction of the 3 rd component of Rodlan was profitable to raise the orientation and the mechanical properties of TLCP in VA fibril in the blend. This presented another example of the 3rd component of Rodlan TLCP playing a role of "orientation providing component."

\section{CONCLUSIONS}

Various characterization techniques were employed to clarify the features of the ternary blends of PBT, Vectra A, and a 3rd component of TLCP of X-7G. The mechanical properties of PBT/VA blend were improved by the addition of a small amount of X-7G TLCP as a 3rd component. Both the mechanical properties and the thermal endurance for the ternary blends of PBT/R5/VA were superior to those of the binary blends of PBT/VA or PBT/X-7G judging from the dynamic viscoelasticities over a wide temperature range. SEM revealed that all the blends studied were phase-separated, but the morphologies of the TLCP were quite different in different parts of the blend samples. The combination of the two kinds of TLCP, VA, and X-7G, was useful to develop regular and longer fibrils with high orientation in the skin part of the injectionmolded samples. The analysis of the WAXD indicated that the introduction of the 3rd component of X-7G TLCP enhanced the degree of the orientation of VA in PBT matrix, and improved the mechanical properties. The results of this study well accorded with the conclusions obtained in our previous paper, where $\mathrm{PC}$ was used as a matrix resin.

Acknowledgments. The authors wish to thank to Toray Co. for supplying PBT sample, to Polyplastics Co. for Vectra A sample and to Unitika Co. for Rodlan samples.

\section{REFERENCES}

1. L-M. Sun, T. Sakamoto, S. Ueta, K. Koga, and M. Takayanagi, Polym. J., 26, 939 (1994).

2. M. Kimura and R.S. Porter, J. Polym. Sci., Polym. Phys. Ed., 22, 1697 (1984).

3. G. Kiss, Polym. Eng. Sci., 27, 410 (1987).

4. T. Harada, A. Ohara, K. Tomari, S. Tonogai, S. Nagai, and K. Yamaoka, The 6th SPE JAPAN RETEC Technical Papers, 1988, p 15.

5. J. Suenaga, E. Fujita, and T. Marutani, Kobunshi Ronbunshu, 48, 573 (1991).

6. A. Zadua, E. Munoz, J.J. Pena, and A. Santmaria, Polymer, 30, 682 (1991).

7. M. Yamauchi, T. Magome, A. Takahara, and T. Kajiyama, Rep. Prog. Polym. Phys. Jpn., 34, 229 (1991). 